\title{
Vers un modèle unifié d'exercice contemporain de la pharmacie en établissement
}

\author{
par James E Tisdale
}

$\mathrm{L}$ orsqu'un patient consulte un médecin où qu'il soit au Canada ou aux États-Unis, il est à peu près certain que la démarche utilisée pour évaluer et diagnostiquer un problème de santé réel ou potentiel sera pratiquement identique à celle des autres médecins, peu importe le lieu et le milieu d'exercice. Bien que différents médecins puissent en arriver à différents diagnostics ou différents plans de traitement pour un même diagnostic, leur processus de décision est en revanche cohérent et essentiellement universel. Cette conformité traduit le fait que les médecins ont recours à un modèle d'exercice établi, cohérent et largement reconnu. Il en va de même pour les dentistes et pour la grande majorité des autres professionnels de la santé.

Par contre, lorsqu' un patient reçoit des soins d'un pharmacien d'hôpital au Canada ou aux États-Unis, il est probable que la démarche utilisée par le pharmacien pour évaluer et diagnostiquer les problèmes pharmacothérapeutiques sera différente, voire qu'elle ne ressemblera en rien à celle d'autres pharmaciens d'hôpitaux. La démarche utilisée par les pharmaciens d'établissements pour évaluer et diagnostiquer un problème pharmacothérapeutique et préparer un plan de traitement varie considérablement d'un praticien à un autre, d'un établissement à un autre, et même d'une unité de soins à une autre dans un même établissement. De plus, il est probable que le rôle particulier du pharmacien au sein de l'équipe de soins de santé variera d'un établissement à un autre et d'une unité de soins à une autre. Plus globalement, les modèles précis de distribution des médicaments et le processus général permettant de s'assurer qu'on remet le bon médicament, au bon patient, au bon moment, varient également d'un établissement à un autre, tout comme les rôles assignés aux techniciens en pharmacie. Même les technologies sous-tendant les systèmes de distribution des médicaments varient considérablement d'un établissement à un autre ${ }^{1,2}$. Bref, la profession de pharmacien, y compris celle des pharmaciens en établissements de santé, n’a pas de modèle d'exercice établi, cohérent et largement reconnu.

Comment expliquer cela? Pourquoi les pharmaciens n'ont-ils pas de modèle d'exercice établi, cohérent et largement reconnu? L'une des explications pourrait bien être que le rôle du pharmacien dans le réseau de la santé n'a pas été clairement défini. Selon moi, le rôle du pharmacien, faisant partie intégrante et indispensable de l'équipe de soins de santé en collaboration interprofessionnelle, est de reconnaitre, de diagnostiquer et de prendre en charge (ou de prévenir) les problèmes pharmacothérapeutiques réels et potentiels et d'être responsable des résultats cliniques associés à la pharmacothérapie. En ce qui a trait au système de distribution des médicaments, je vois le pharmacien y jouer principalement un rôle de superviseur et les techniciens en pharmacie, à l'aide de la technologie appropriée, y assumer particulièrement la responsabilité des fonctions au sein du système. En revanche, j'ai le sentiment que de nombreux pharmaciens et autres professionnels de la santé ne seront pas nécessairement d'accord avec moi et qu'ils proposeraient une définition différente du rôle du pharmacien au sein du système de santé. Si nous ne pouvons pas nous entendre sur une définition commune de notre rôle, alors comment pouvons-nous définir un modèle d'exercice de la pharmacie approprié pour justement jouer ce rôle?

Pourquoi est-il important que la médecine, la dentisterie et, j'ajouterai, la pharmacie, aient leur propre modèle d'exercice établi, cohérent et largement reconnu? Pourvu que les problèmes pharmacothérapeutiques de chaque patient soient adéquatement pris en charge, la démarche utilisée par le pharmacien pour arriver à ses fins revêt-elle une importance? Je crois qu'en l'absence d'un modèle d'exercice établi et invariablement efficace, il est impossible de reconnaitre, de diagnostiquer et de prendre en charge (ou de prévenir) de façon systématique et précise les problèmes pharmacothérapeutiques réels et potentiels et, par conséquent, d'améliorer les résultats cliniques associés à la pharmacothérapie. Dans le domaine de la pharmacie, nous ignorons toujours quel est le modèle d'exercice le plus efficace pour améliorer les résultats cliniques, ce qui constitue un terreau fertile pour la recherche sur l'exercice de la pharmacie et l'efficacité relative. Un modèle d'exercice efficace et cohérent permettrait probablement d'asseoir et d'affirmer l'importance des pharmaciens auprès des patients et des autres professionnels de la santé avec lesquels nous collaborons à la prestation des soins, car cela démontrerait de façon plus systématique qui nous sommes, ce que nous faisons et la raison 
pour laquelle notre travail est si important dans la prestation des soins aux patients.

Des modèles d'exercice de la pharmacie applicables à tous les pharmaciens, peu importe le milieu dans lequel ils exercent, ont déjà été proposés. Plus particulièrement, le modèle des soins pharmaceutiques a été proposé par Cipolle et ses collaborateurs dans les années $1990^{3}$. Toutefois, bien que ce modèle d'exercice ait reçu beaucoup d'attention et nourri beaucoup de discussions, et que quelques-uns aient adopté certains de ses éléments, il n’a jamais été largement accepté ni mis en œuvre dans son intégralité par la majorité des praticiens.

Dans une certaine mesure, l'évolution de l'exercice de la pharmacie hospitalière s'enchâsse dans les buts du projet SCPH 2015 de la Société canadienne des pharmaciens d'hôpitaux $x^{4}$. Les buts de SCPH 2015 sont notamment d'accroître le degré d'intervention des pharmaciens auprès des patients hospitalisés et non hospitalisés afin d'assurer une utilisation optimale des médicaments, d'étendre l'application du principe des décisions fondées sur les preuves à la pratique clinique quotidienne des pharmaciens des établissements de santé dans le but d'améliorer la pharmacothérapie, d'accroître le rôle joué par les départements de pharmacie des établissements de santé dans l'amélioration de l'utilisation sécuritaire des médicaments, d'étendre l'application efficace des technologies dans les départements de pharmacie des établissements de santé pour améliorer l'utilisation sécuritaire des médicaments et d'accroître le degré d'intervention des départements de pharmacie des établissements de santé dans la mise en œuvre d'initiatives de santé publique. Malgré que l'atteinte de ces buts puisse dans certains établissements être réalisée en changeant le modèle d'exercice, le projet SCPH 2015 ne préconise ni n'encourage le développement d'un modèle d'exercice de la pharmacie hospitalière établi, cohérent et largement reconnu.

La détermination d'un modèle optimal d'exercice contemporain de la pharmacie en établissement est aujourd'hui un enjeu crucial pour l'American Society of Health-System Pharmacists (ASHP) et sa Fondation, qui, en 2008, ont mis de l'avant le projet de modèle d'exercice de la pharmacie (Pharmacy Practice Model Initiative [PPMI]). Le but du PPMI est « d'améliorer sensiblement la santé et le bien-être des patients en élaborant et en disséminant un modèle d'exercice futuriste qui favorise l'utilisation la plus efficace des pharmaciens comme fournisseurs de soins directs aux patients " ${ }^{5}$. Le modèle d'exercice finalement élaboré vise à "refléter l'évolution de nombreux aspects de l'exercice de la pharmacie dans les établissements de santé au cours des 50 dernières années ", et cela comprend une politique sur l'utilisation des médicaments et la sélection des produits, la distribution des médicaments, l'exercice de la pharmacie clinique, les rôles des techniciens en pharmacie, les rôles des pharmaciens en tant que chefs de file au sein des organisations, la conformité à la pratique fondée sur les normes, la réaction aux mouvements de qualité et de sécurité relatives à l'utilisation des médicaments aux États-Unis et la portée de la technologie ${ }^{6}$. Les principaux objectifs du PPMI sont de créer un cadre pour un modèle d'exercice de la pharmacie qui
" assure la prestation sécuritaire, efficace, efficiente, responsable et fondée sur des données probantes des soins pour tous les patients dans les établissements de santé "; de déterminer les services qui devraient être fournis de façon cohérente par les services de pharmacie hospitaliers; de repérer les technologies émergentes favorisant la mise en ouvre du modèle d'exercice; de concevoir un gabarit pour le modèle d'exercice; et de mettre de l'avant des mesures permettant de générer des changements permanents dans le modèle d'exercice de la pharmacie en établissement ${ }^{7}$.

Le PPMI a débuté par un sommet sur le modèle d'exercice de la pharmacie qui s'est tenu du 7 au 9 novembre 2010 à Dallas, au Texas. Au cours de ce sommet, plus de 100 leaders d'opinion se sont réunis pour déterminer les résultats clés attendus de la conception de nouveaux modèles d'exercice ${ }^{5}$. La pharmacie hospitalière canadienne était représentée par un pharmacien d'hôpital du Canada et un représentant de la SCPH. Un consensus a été obtenu sur plusieurs idées spécifiques, notamment : le besoin de changements dans la façon dont les pharmaciens d'établissements, les techniciens et la technologie sont utilisés; la responsabilité des services de pharmacie à contribuer à la santé financière globale de leurs établissements respectifs; l'exigence par les dirigeants des hôpitaux d'une exploitation plus efficiente des services de pharmacie dans les 5 à 10 prochaines années; l'assignation des fonctions de préparation habituelle des produits stériles aux techniciens en pharmacie ayant les qualifications et la formation adéquates; et la nécessité pour les pharmaciens de se percevoir comme des professionnels et d'agir comme des professionnels en tout temps 5 . Les prochaines étapes du PPMI seront notamment la mise en route d'une campagne de marketing social pour communiquer à un large auditoire les comptes rendus et les résultats du sommet. De plus, des subventions pour des projets expérimentaux portant principalement sur l'influence du changement de modèle d'exercice sur l'utilisation des médicaments seront offertes par la Fondation pour la recherche et l'éducation de l'ASHP

La détermination et la mise en ouvre d'un modèle d'exercice de la pharmacie cohérent et largement reconnu sont un objectif louable. Mais dans quelle mesure la conception d'un modèle de pratique futuriste qui favorise l'utilisation la plus efficace des pharmaciens comme fournisseurs de soins directs aux patients aux États-Unis influera-t-elle sur l'exercice de la pharmacie hospitalière au Canada? Cela reste à voir. Certes, il y a des différences considérables dans la prestation des soins de santé entre nos deux pays. En revanche, malgré ces différences, les modèles d'exercice des médecins et des dentistes au Canada et de leurs homologues aux États-Unis ne sont pas sensiblement différents. Les pharmaciens et les services de pharmacie audacieux des hôpitaux du Canada pourront saisir des occasions d'expérimenter de nouveaux modèles d'exercice de la pharmacie et de mener des recherches comparant l'efficacité des divers modèles. Les pharmacies d'hôpitaux d'Amérique du Nord ont donc la chance de se redéfinir, de définir et d'affirmer le rôle des pharmaciens au sein des équipes de soins de santé et d'établir un 
modèle d'exercice cohérent et largement reconnu qui, en définitive, améliorera les résultats cliniques associés à la pharmacothérapie des patients. C'est le moment où jamais!

[Traduction par l'éditeur]

\section{Références}

1. Bussières JF. Services cliniques de pharmacie. Dans : Babich M, Bussières JF Hall KW, Harding J, Johnson N, Lefebvre P, et al., rédacteurs. Rapport 2007-2008 sur les pharmacies hospitalières canadiennes. Eli Lilly; 2008. p. 4-24. Publié au www.lillyhospitalsurvey.ca/hpc2/content/2008_ report/ 2007-2008F\%20full.pdf. Consulté le 11 janvier 2011.

2. Harding J. Systèmes de distribution des médicaments. Dans : Babich M, Bussières JF, Hall KW, Harding J, Johnson N, Lefebvre P, et al., rédacteurs. Rapport 2007-2008 sur les pharmacies hospitalières canadiennes. Eli Lilly; 2008. p. 25-37. Publié au www.lillyhospitalsurvey.ca/hpc2/content/ 2008_report/2007-2008F\%20full.pdf. Consulté le 11 janvier 2011.

3. Cipolle RJ, Strand LM, Morley PC. Pharmaceutical care practice. New York (NY) : McGraw-Hill; 1998.

4. SCPH 2015 : Buts et objectifs (document révisé le 19 mai 2008). Ottawa (ON): Société canadienne des pharmaciens d'hôpitaux. Publié au www. cshp.ca/dms/dmsView/1_SCPH-2015---Final-jan\%2710.pdf. Consulté le 11 janvier 2011.

5. Thompson CA. Pharmacy Practice Model Initiative finishes consensusbuilding process. Am J Health Syst Pharm 2010;67(24):2078,2080,2082.

6. Overview/rationale. In: Pharmacy Practice Model Initiative [site Web]. Bethesda (MD): American Society of Health-System Pharmacists. Publié au www.ashp.org/PPMI/AboutPPMI/Overview.aspx. Consulté le 11 janvier 2011
7. Objectives. In: Pharmacy Practice Model Initiative [site Web]. Bethesda (MD): American Society of Health-System Pharmacists. Publié au www. ashp.org/PPMI/AboutPPMI/Objectives.aspx. Consulté le 11 janvier 2011.

8. Activities. In: Pharmacy Practice Model Initiative [site Web]. Bethesda (MD): American Society of Health-System Pharmacists. Publié au www. ashp.org/PPMI/AboutPPMI/Activities.aspx. Consulté le 11 janvier 2011.

James E Tisdale, Pharm. D., est professeur à la Faculté de pharmacie de I'Université Purdue et professeur associé de l'École de médecine de I'Université d'Indiana, à Indianapolis, en Indiana, aux États-Unis. II est également rédacteur adjoint du JCPH.

\section{Adresse de correspondance :}

Dr James E Tisdale

College of Pharmacy

Purdue University

W7555 Myers Building, WHS

1001 West 10th Street

Indianapolis IN 46202 USA

Courriel : jtisdale@iupui.edu

\section{ON THE FRONT COVER}

\section{"Almost Without Surface" Abraham Lake, Clearwater County, Alberta}

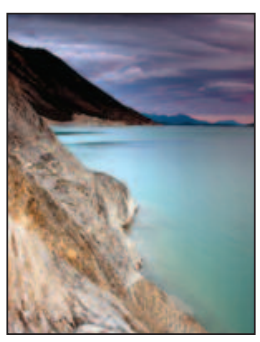

Abraham Lake is the largest artificial lake in Alberta. Named after Silas Abraham, a Stoney First Nations guide who spent his life in the area, the lake was created in 1972 when the Bighorn hydroelectric dam was commissioned on the North Saskatchewan River. This photograph was taken just after sunrise on October 30, 2009, by Jim Dobie, husband of
CSHP member Terri Schindel. The photographer used a Canon 40D camera, with Sigma 10-20 lens at $17 \mathrm{~mm}$ (f/16, shutter speed 2.5 seconds).

The CJHP would be pleased to consider photographs featuring Canadian scenery taken by CSHP members for use on the front cover of the journal. If you would like to submit a photograph, please send an electronic copy (minimum resolution $300 \mathrm{dpi}$ ) to Colleen Drake at cdrake@cshp.ca. 